\title{
Risk of Obstetric Anal Sphincter Injury with Fundal Pressure in Second Stage of Labour; Transperineal Ultrasound Study
}

\author{
Hande Öncï ${ }^{1}$, Serdar Aydın², and Çağrı Arıŏlu Aydın ${ }^{3}$ \\ ${ }^{1}$ Bezmialem Vakif University \\ ${ }^{2}$ Koc University School of Medicine \\ ${ }^{3}$ Medical Park Hospital
}

September 17, 2020

\begin{abstract}
Objective: The aim of this study was to evaluate the effect of using uterine fundal pressure during the second stage of delivery on obstetric anal sphincter injury (OASI), among primiparous women using three-dimensional transperineal ultrasonography. Design: Case control study Setting: Tertiary Urogynecology Unit Population: Nulliparous women with term, singleton, cephalic presentation gestation delivered with fundal pressure in second stage of labour. Main Outcome Measure: Complete, incomplete IAS and EAS defect in transperineal tomographic ultrasound imaging Method: A total of 73 women who had their first vaginal birth were included in the study, 37 of them applied fundal pressure and 36 of them delivered spontaneously without fundal pressure. Tomographic ultrasound imaging with 3D transperineal assessment was performed within $48 \mathrm{~h}$ of delivery, IAS and EAS defect were determined. Results: Five (13.5\%) women in the fundal pressure group, 7 (20\%) women in the control group had complete EAS $(\mathrm{p}=0.4)$. Complete IAS was observed in $1(2.7 \%)$ women in the fundal pressure group and $2(5.7 \%)$ women in the control group $(\mathrm{p}=0.5)$. Half-moon sign was observed in 1 woman in both groups $(\mathrm{p}=0.9)$. The rate of other signs were similar in both groups. Multivariate regression models revealed that none of, age, episiotomy, length of second stage of labour, fundal pressure application status and number were independent predictor of complete IAS or EAS defect. Conclusions: Fundal pressure during the second stage of delivery is not cause increase in rate of OASI detected with ultrasonography.
\end{abstract}

\section{Tweetable abstract}

Fundal pressure during the second stage of delivery is not cause increase in rate of OASI detected with ultrasonography.

Keywords: fundal pressure, obstetric anal sphincter injury, pelvic floor, transperineal ultrasound, vaginal delivery

Clinical Trial Registration: NCT03752879

\section{Introduction}

Obstetric anal sphincter injuries (OASI) include third and fourth degree perineal tears and have a major impact on quality of life by resulting in chronic anal incontinence, dyspareunia or perineal pain. OASI may complicate 0.3 to 6 percent of vaginal deliveries related to multiple fetal, maternal and obstetrics factors. ${ }^{1-3}$ Clinical management of second stage of labour and effectiveness of preventive measures for severe perineal tears are controversial. Operative vaginal delivery is related with increased risk of OASI, reported incidence up to $27 \%$ in primiparous women delivering by forceps delivery without an episiotomy. ${ }^{4}$ Also the risk of OASI is increased approximately 3 times $(\mathrm{OR}=2.99)$ times vacuum-assisted delivery. ${ }^{5}$ Increased maternal age, fetal macrosomia and persisted fetal occipital posterior position are the other risk factors. ${ }^{6,7}$ 
In clinical practice uterine fundal pressure (the Kristeller maneuver) commonly applied in second stage of delivery to accelerate the birth in cases of fetal distress, dystocia, failure to progress in the second stage of delivery, maternal unwillingness to strain due to exhaustion. ${ }^{8}$ Benefits, reliability and complications of this maneuver are uncertain and real usage frequency may be underestimated owing to medico-legal aspects and adverse outcomes. ${ }^{9}$ The reported frequency of the manual fundal pressure ranges from $4 \%$ to $23 \%$ and is frequently used in settings where instrumental deliveries, are not readily available, or cannot be performed because of professional staff shortage. ${ }^{10}$

Limited availability of endo-anal imaging systems and the development of advanced high-frequency curved array transducers, three-dimensional transperineal ultrasonography (3D-TPU) has attracted attention for its effectiveness in evaluating anal sphincter injury. Three dimensional TPU allows access to the 3 orthogonal planes at same time, tomographic image showing entire sphincter, with minimal patient discomfort and low cost. ${ }^{11}$ Exoanal 3D-TPU imaging have validity and repeatability for anal sphincter injuries in parallel to improving of image quality and software of 3D ultrasound systems. ${ }^{12}$

Identification of avoidable risk factors of OASI is important to avoid unnecessary interventions which may cause long term morbidity. Therefore, the aim of this study was to evaluate the effect of using uterine fundal pressure during the second stage of delivery on OASI among primiparous women using 3D-TPU exoanal imaging.

\section{Materials and Methods}

This case-control study (NCT03752879) was conducted at the urogynecology unit of the University Hospital in Istanbul, Turkey. The study protocol was approved by the instutional ethical committee (IRB No: 2019$7 / 22$ ). Written informed consent for the study was obtained at hospitalization of nulliparas, who were the nominee for vaginal delivery. The study population included nulliparous women over 18 years old, with 37 and 42 weeks of singleton cephalic presentation gestation. Potential participants were excluded if they were multiparous, had a history of instrumental delivery, chronic intestinal disease (Crohn's disease, ulcerative colitis), anal incontinence, surgery to anal canal.

Oxytocin and a prostaglandin E2 pessary was used for induction of labour and oxytocin was used for augmentation during the first and second stage of delivery if necessary. None of the patients received spinal/epidural analgesia and a mediolateral episiotomy was performed if necessary. For the fundal pressure group, pressure was applied manually by an obstetrician during the second stage of delivery while the fetal heart rate was being monitored. The obstetrician pressed on the uterine fundus at a $30-45^{\circ}$ angle to the maternal spine, in the direction of the pelvis, and a longitudinal direction with each uterine contraction until delivery of the fetal head.

\section{D-TPU assessments}

The same investigator (H.N.O), blinded to clinical and fundal pressure data, performed all the ultrasound volume acquisitions within 48 h of delivery, using a GE Voluson E6 (GE Penta Healthcare) with a 2-8-MHz convex volume, real-time $4 \mathrm{D}$ transducer. A thin film covered $4 \mathrm{D}$ probe placed on the perineum transversely, with light touch oriented postero-inferiorly to anal canal. Pelvic floor imaging settings were chosen, acquisition angle was set to $60^{\circ}$ or $70^{\circ}$, the image quality settings and harmonics increased to maximum for optimization of resolution.

Tomographic ultrasound imaging (TUI) on maximum pelvic floor muscle contraction was used to assess the entire external and internal anal sphincter as described by Dietz et al. ${ }^{11}$ Six slices, at $2 \mathrm{~mm}$ slice intervals, were created in the axial plane, from at the level of the subcutaneous portion of EAS to at the level of anorectal junction, cranial to IAS. The AS was evaluated in 6 central slices and sphincter defect was diagnosed as a discontinuity of the ring shape of the EAS and IAS, appearing hypoechoic in IAS relative to the EAS and hyperechoic relative to the IAS in EAS. A complete AS defect has been defined as a defect of $30^{\circ}$ or greater in circumference of AS in at least 3 slices (Figure 1). Incomplete defect described when less than 3 and/or partial discontinuity occurs in EAS or IAS (Figure 2). Other signs; thinning of EAS and/or IAS, thinning 
of EAS and/or IAS, thickening of the IAS opposite the defect, "halfmoon sign" were used indicate defect of AS (Figure 3). Half moon sign describes thickening of the IAS opposite the rupture site, with thinning or interruption in the area of repair. ${ }^{13}$ Analysis of stored volumes was conducted offline using 4D VIEW version 10.2; GE Healthcare, London, UK. An interobserver test-retest series was conducted for complete IAS and EAS defect on the volumes recorded for the first 20 patients recruited for this study.

\section{Rectal Examination}

In order to evaluate the anal sphincter pressure of the patients, we used the digital rectal examination scoring system (DRESS) by performing a rectal examination after the ultrasound acquisition was completed. The DRES System uses an analog scale of 0 to 5 . During rectal examination, separate numbers are assigned to the resting and maximum squeeze pressure. A score of 3 is normal. A rest score of 5 indicates very high pressures and a tight anal canal, while a score of 0 indicates an open anal canal at rest with the hips split. A squeeze score of 5 indicates a very strong squeeze that was almost painful to the exam, while a score of 0 indicates no increase in pressure noticeable from resting with maximum patient effort. The DRESS score is a validated system which is correlated very well with manometer pressures for resting pressure and squeeze pressure. $^{14}$

After the vaginal delivery, the participants were divided into two groups: the fundal pressure group included women where the fundal pressure maneuver was applied $(n=37)$; and the control group included women who delivered spontaneously without fundal pressure $(n=36)$. Demographic and obstetric data including age, body mass index (BMI), fetal height, fetal weight, fetal bi-parietal diameter (BPD), head circumference (HC) and duration of the first stage of delivery. The duration of the second stage of delivery, defined as the period from full dilation of the cervix until complete expulsion of the baby, was noted. Furthermore, data on the need for episiotomy, the number of fundal pressure trials, labour complications and 3D-TPU findings were documented.

\section{Statistical analysis}

Sample size calculation was performed by using data from van Bavel's study, which evaluates the risk of OASI: the true risk of OASI in primiparous women with mediolateral episiotomy is $3 \%$ and $26 \%$ in instrumental delivery. ${ }^{4}$ A sample size of 33 for each group was calculated based on these data and a one-sided $t$-test $(p<0.05)$ was estimated to have $80 \%$ power to reject the null hypothesis that uterine fundal pressure does not increase the risk of OASI as much as forceps delivery. Statistical analysis was performed after normality testing (histogram analysis and/or Kolmogorov-Smirnov test) using IBM SPSS, version 21 (IBM Inc., Armonk, NY). Student's t-test was used for the comparison of normally distributed variables, while the Mann-Whitney U-test was used for non-parametric variables, and Fisher's exact tests were used to compare categorical variables. Variables were then considered jointly in a multivariable stepwise forward multivariable logistic regression model to predict OASI.

\section{Results}

During the study period, a total of 73 women were included in the study, 37 of them applied fundal pressure and 36 of them delivered spontaneously without fundal pressure. The mean age of fundal pressure group was $25.3 \pm 3.4$, and the mean age of the control group was $27.7 \pm 4.8(\mathrm{p}=0.01)$. The groups were similar to each other with regard to BMI, fetal weight, fetal BPD, fetal HC, and duration of the first stage of delivery. The duration of the second stage of delivery was significantly longer in the fundal pressure group compared to the controls (37.3 \pm 8.6 min vs. $57.3 \pm 19.2 \mathrm{~min}$, respectively; $p<0.0001)$. Baseline and delivery characteristics of groups presented in Table 1. No statistical difference was observed between the two groups in terms of labour induction, PGE2 and oxytocin application. Elective episiotomy was applied to 32 (86.5\%) of 37 women who had fundal pressure, and $15(42.9 \%)$ of 36 women in the control group $(\mathrm{p}<0.0001)$. There were $5(13.5 \%)$ women delivered with spontonaeus perineal tear in the fundal pressure group and $16(45.7 \%)$ women in the control group $(\mathrm{p}=0.003)$. In the control group, 4 patients $(11.4 \%)$ delivered without episiotomy or any perineal tear $(\mathrm{p}=0.03)$. 
Five (13.5\%) women in the fundal pressure group, $7(20 \%)$ women in the control group had complete EAS (p $=0.4)$ (Table 2). Complete IAS was observed in $1(2.7 \%)$ women in the fundal pressure group and $2(5.7 \%)$ women in the control group $(\mathrm{p}=0.5)$. Half-moon sign was observed in 1 woman in both groups $(\mathrm{p}=0.9)$. The rate of other signs were similar in both groups.

Multivariate regression models revealed that none of, age, episiotomy, length of second stage of labour, fundal pressure application status and number were independent predictor of complete IAS or EAS defect.

\section{Discussion}

According to results of our study we suggested that fundal pressure at second stage of labour did not cause an increase in anal sphincter injury, contrary to expectations. Although the study designs are different, Cosner et al. stated that the second stage of labour was longer in the group in which fundal pressure was applied, and the incidence of third and fourth degree perineal lacerations was higher than the group without fundal pressure. ${ }^{15}$ Also our second stage of labour length was significantly longer in fundal pressure group. We know that Kristeller maneuver applied commonly in long lasting deliveries and difference in second stage of labour in our results is compatible with literature. ${ }^{16,17}$ In another studies, fundal pressure is associated with shortened second stage and increased risk of severe perineal lacerations. ${ }^{18}, 19$ The inconsistency between our results and Cosner's pilot study can be explained by uncertainty whether the accompanying episiotomy was median mediolateral and high rate $(29.4 \%)$ of OASI in fundal pressure group. ${ }^{15}$ In others, it was not specified whether severe perineal injuries were OASI or third/fourth degree perineal tears. ${ }^{18,19}$

Various methods have been investigated to reduce the risk of perineal tears during delivery. These methods include perineal massage, manual perineal support, warm compresses, limited use of episiotomy, and delayed straining. However the effect of mediolateral episiotomy on OASI in spontaneous vaginal deliveries is not clear. A recent meta-analysis concluded that mediolateral episiotomy can reduce OASI and should not be prevented, especially in nulliparous women. ${ }^{20}$ In an endoanal ultrasound study with 60 participants which evaluates impact of mediolateral episiotomy on incidence of obstetrical anal sphincter injury, authors suggested that mediolateral episiotomy does not seem to be protective against clinical or sonographic diagnosed OASIS even when episiotomy technique is considered. ${ }^{21}$ In studies comparing mediolateral episiotomy with medial episiotomy, it is clear that mediolateral episiotomy has a lower risk of obstetric injury compared to median episiotomy. ${ }^{20,22}$ If an episiotomy is required, mediolateral episiotomy has been shown to be a protective intervention for OASI, especially in nulliparous women. ${ }^{23-25}$ Also the use of mediolateral episiotomy has a highly protective effect on the incidence of OASI during operative vaginal delivery. ${ }^{6}$ We thought that the application of liberal mediolateral episiotomy with over $45^{\circ}$ from midline might have a protective effect on the anal sphincter in patients undergoing fundal pressure in our study. ${ }^{26}$

There are studies evaluating the effect of the Kristeller maneuver on the pelvic floor muscles rather than the studies evaluating the effect on the anal sphincter. Yousef et al., in their study on the effect of the Kristeller maneuver on pelvic floor muscles, showed that the application of fundal pressure in the second stage of labour was associated with more than twice the risk of levator ani muscle avulsion in women with a first vaginal delivery. ${ }^{27}$ In our recent study about pelvic floor damage and fundal pressure Fundal pressure during the second stage of delivery is associated with a higher risk of levator ani muscle defect and loss of anterior vaginal wall support. ${ }^{28}$ In these study, similar to our study, the duration of the second stage of labour was significantly longer in the fundal compression group, and episiotomy was performed more frequently in the fundal compression group.

\section{Strengths and limitations}

To the best of our knowledge, this case control study was the first to evaluate the association between fundal pressure and OASI immediately after birth with TPUS. Homogeneity of obstetric applications, documentation of fundal pressure indications and numbers were the strength of the present study. Major limitation of this study was the relatively small sample size. Lack of a standardized protocol for the application of fundal pressure and multiplicity of practitioners of fundal pressure were the another limitations. More frequent use of mediolateral episiotomy in fundal pressure group may obscure the effect of fundal pressure on anal 
sphincter.

\section{Conclusion}

Fundal pressure is commonly considered before operative vaginal birth or cesarean section in the case of a prolonged second stage of delivery or fetal distress especially in settings where instrumental deliveries, are not readily available, or cannot be performed because of professional staff shortage. It is important to objectively evaluate safety of fundal pressure on anal sphincter injury. According to our results, we concluded that fundal pressure during the second stage of delivery is not associated with a higher risk of OASI. Further well designed, large prospective studies are needed to estimate the long-term anatomical and functional outcomes of fundal pressure on anal sphincter.

\section{Disclosure of interests}

The authors report no conflicts of interest. The authors alone are responsible for the content and writing of this paper

\section{Funding}

None

\section{Acknowledgements}

None

\section{Contribution to authorship}

HNO and SA conceived and designed the study. HNO and SA formulated the research questions. CA and SA were responsible for data management and statistical analysis. HNO and SA analyzed the data. CA and SA drafted the paper, which was revised and approved by all authors. CA, HNO and SA contributed by revising the manuscript and providing important input.

\section{Details of ethics approval}

The Ethical Review Board at the Bezmialem Vakif University of Istanbul (IRB No: 2019-7/22) approved the study. Date of approval 03 April 2019.

\section{References}

1. Selmer-Olsen T, Nohr EA, Tappert C, Eggebo TM. Incidence and risk factors for obstetric anal sphincter ruptures, OASIS, following the introduction of preventive interventions. A retrospective cohort study from a Norwegian hospital 2012-2017. Sex Reprod Healthc. 2019;22:100460.

2. Farrar D, Tuffnell DJ, Ramage C. Interventions for women in subsequent pregnancies following obstetric anal sphincter injury to reduce the risk of recurrent injury and associated harms. Cochrane Database Syst Rev. 2014 (11):CD010374. doi(11):CD010374.

3. Gurol-Urganci I, Cromwell DA, Edozien LC, Mahmood TA, Adams EJ, Richmond DH, et al. Thirdand fourth-degree perineal tears among primiparous women in England between 2000 and 2012: time trends and risk factors. BJOG. 2013;120(12):1516-25.

4. van Bavel J, Hukkelhoven CWPM, de Vries C, Papatsonis DNM, de Vogel J, Roovers JWR, et al. The effectiveness of mediolateral episiotomy in preventing obstetric anal sphincter injuries during operative vaginal delivery: a ten-year analysis of a national registry. Int Urogynecol J. 2018;29(3):407-13.

5. Jangö H, Langhoff-Roos J, Rosthøj S, Sakse A. Modifiable risk factors of obstetric anal sphincter injury in primiparous women: a population-based cohort study. Am J Obstet Gynecol. 2014;210(1):59.e159.e596.

6. de Leeuw JW, de Wit C, Kuijken JP, Bruinse HW. Mediolateral episiotomy reduces the risk for anal sphincter injury during operative vaginal delivery. BJOG. 2008;115(1):104-8.

7. Meister MR, Cahill AG, Conner SN, Woolfolk CL, Lowder JL. Predicting obstetric anal sphincter injuries in a modern obstetric population. Am J Obstet Gynecol. 2016;215(3):310.e1-7. 
8. Simpson KR, Knox GE. Fundal pressure during the second stage of labour. MCN Am J Matern Child Nurs. 2001;26(2):64-71. doi:10.1097/00005721-200103000-00004

9. Merhi ZO, Awonuga AO. The role of uterine fundal pressure in the management of the second stage of labour: a reappraisal. Obstet Gynecol Surv. 2005;60(9):599-603. doi:10.1097/01.ogx.0000175804.68946.ac

10. Hofmeyr GJ, Vogel JP, Cuthbert A, Singata M. Fundal pressure during the second stage of labour. Cochrane Database Syst Rev. 2017;3(3):CD006067. Published 2017 Mar 7. doi:10.1002/14651858.CD006067.pub3

11. Dietz HP. Exoanal Imaging of the Anal Sphincters. J Ultrasound Med. 2018;37(1):263-280. doi:10.1002/jum.14246

12. Turel Fatakia F, Subramaniam N, Bienkiewicz J, Friedman T, Dietz HP. How repeatable is assessment of external anal sphincter trauma by exoanal 4D ultrasound?. Ultrasound Obstet Gynecol. 2019;53(6):836-840. doi:10.1002/uog.20175

13. Valsky DV, Messing B, Petkova R, Savchev S, Rosenak D, Hochner-Celnikier D, Yagel S. Postpartum evaluation of the anal sphincter by transperineal three-dimensional ultrasound in primiparous women after vaginal delivery and following surgical repair of third-degree tears by the overlapping technique. Ultrasound Obstet Gynecol 2007; 29: 195-204

14. Bruce A.Orkin,M.D. SvetlanaB.Sinykin,B.A. PatriciaC.Lloyd,Sc.M The Digital Rectal Examination Scoring System (DRESS) Dis Colon Rectum 2010; 53: 1656 -1660

15. Cosner KR. Use of fundal pressure during second- stage labour. A pilot Study. J Nurse Midwif. 1996;41: $334-337$.

16. Cuerva MJ, Tobias P, Espinosa JA, et al. Intrapartum ultrasound prior to Kristeller maneuver: an observa- tional study. J Perinat Med. 2015;43:171-175.

17. Malvasi A, Montanari Vergallo G, Tinelli A, et al. Can the intrapartum ultrasonography reduce the legal liability in distocic labour and delivery? J Matern Fetal Neonatal Med. 2018;31:1108-1109

18. Moiety FM, Azzam AZ. Fundal pressure during the second stage of labour in a tertiary obstetric center: a prospective analysis. J Obstet Gynaecol Res 2014; 40: 946-953

19. Matsuo K, Shiki Y, Yamasaki M, Shimoya K. Use of uterine fundal pressure maneuver at vaginal delivery and risk of severe perineal laceration. Arch Gynecol Obstet 2009; 280: 781-786.

20. Verghese TS, Champaneria R, Kapoor DS, Latthe PM. Obstetric anal sphincter injuries after episiotomy: Systematic review and meta-analysis. Int Urogynecol J. 2016;27:1459-1467.

21. Drusany Staric K, Lukanovic A, Petrocnik P, Zacesta V, Cescon C, Lucovnik M. Impact of mediolateral episiotomy on incidence of obstetrical anal sphincter injury diagnosed by endoanal ultrasound. Midwifery. 2017;51:40-43. doi:10.1016/j.midw.2017.05.008

22. Jiang H, Qian X, Carroli G, Garner P. Selective versus routine use of episiotomy for vaginal birth. Cochrane Database Syst Rev. 2017;(2):CD000081.

23. Coats PM, Chan KK, Wilkins M, et al. A comparison between midline and mediolateral episiotomies. Br J Obstet Gynaecol. 1980;87:408-412.

24. Aukee P, Sandstrom H, Kairaluoma MV. The role of mediolateral episioto- my during labour: analysis of risk factors for obstetric anal sphincter tears. Acta Obstet Gynecol Scand. 2006;85(7):856-860.

25. Poen AC, Felt-Bersma RJ, Dekker GA, et al. Third degree obstetric perineal tears: risk factors and the preventative role of mediolateral episiotomy. Br J Obstet Gynaecol. 1997;104:563-566.

26. Eogan M, Daly L, O'Connell PR, et al. Does the angle of episiotomy affect the incidence of anal sphincter injury? BJOG. 2006;113:190-194.

27. Youssef A, Salsi G, Cataneo I, et al. Fundal pressure in second stage of labour (Kristeller maneuver) is associated with increased risk of levator ani muscle avulsion. Ultrasound Obstet Gynecol. 2019;53(1):95-100. doi:10.1002/uog.19085

28. Takmaz T, Aydın S, Gorchiyeva İ, Karasu AFG. The usual suspect: cross-sectional study of fundal pressure at second stage of delivery and the association with pelvic floor damage. Int Urogynecol J. 2020;10.1007/s00192-020-04523-x. doi:10.1007/s00192-020-04523-x

Figure 1: Imaging of the anal sphincters with 3D transperineal ultrasound. The white stars indicates a discontinuity in the internal anal sphincter in 3 slices that corresponds to a complete IAS defect.Figure 2: 
Imaging of the anal sphincters with $3 \mathrm{D}$ transperineal ultrasound. The black arrows indicates a discontinuity in the internal anal sphincter in 2 slices that corresponds to a incomplete IAS defect.Figure 3: Thinning of internal anal sphincter indicated with white arrows.

\begin{tabular}{|c|c|c|}
\hline Characteristics & Fundal pressure group $(\mathrm{n}=37)$ & Control group $(n=36)$ \\
\hline Age,years $($ mean \pm SD) & $25.3 \pm 3.4$ & $27.7 \pm 4.8$ \\
\hline $\mathrm{BMI}, \mathrm{kg} / \mathrm{m}^{2}(\operatorname{mean} \pm \mathrm{SD})$ & $28.7 \pm 3.3$ & $28.6 \pm 5.1$ \\
\hline Fetal weight, $g($ mean $\pm S D)$ & $3321 \pm 393$ & $3225 \pm 380$ \\
\hline Fetal height, cm (mean \pm SD $)$ & $50.2 \pm 2.1$ & $49.3 \pm 2.2$ \\
\hline Fetal BPD, mm (mean \pm SD) & $91.7 \pm 3,5$ & $85.4 \pm 9.70$ \\
\hline Fetal HC, mm $($ mean $\pm \mathrm{SD})$ & $321.7 \pm 18.8$ & $317.5 \pm 10.3$ \\
\hline Duration of the first stage of labour, min $($ mean $\pm \mathrm{SD})$ & $549 \pm 379$ & $501 \pm 388$ \\
\hline Duration of the second stage of labour,min $($ mean $\pm \mathrm{SD})$ & $57.3 \pm 19.2$ & $37.3 \pm 8.6$ \\
\hline Induction of Labour, n (\%) & $22(59.5)$ & $21(58.3)$ \\
\hline $\mathrm{PGE}_{2}$ Induction, $\mathrm{n}(\%)$ & $7(18,9)$ & $8(22.2)$ \\
\hline Oxytocin administration, $\mathrm{n}(\%)$ & $15(40.5)$ & $13(36.1)$ \\
\hline Spontaneous Perineal Tear, n (\%) & $5(13.5)$ & $16(44.4)$ \\
\hline Episiotomy, n (\%) & $32(86.5)$ & $15(41.7)$ \\
\hline No tear, n $(\%)$ & 0 & $4(\% 11.1)$ \\
\hline Complication, n (\%) & $3(8.1)$ & $2(5.6)$ \\
\hline Hematoma, n (\%) & $2(5.4)$ & $2(5.6)$ \\
\hline Extended Episiotomy n (\%) & $2(5.4)$ & - \\
\hline Postpartum prolapse, n (\%) & $4(10.8)$ & $2(5.6)$ \\
\hline Postpartum anal incontinece, n (\%) & $2(5.4)$ & $1(2.8)$ \\
\hline
\end{tabular}

Table 1 . Baseline and delivery characteristics of groups. BMI; body mass index, BPD; biparietal diameter, $\mathrm{HC}$; head circumference, $\mathrm{PGE}_{2}$; Prostoglandin E2,

Bolding indicates statistical significance.

* Student t test.

** Mann-Whitney U test.

$+\mathrm{X}^{2}$ test

++ Fisher exact test.

\begin{tabular}{llll}
\hline & Fundal pressure group $(\mathbf{n}=\mathbf{3 7})$ & Control group $(\mathbf{n}=\mathbf{3 6})$ & $\mathrm{P}$ value \\
\hline Complete EAS Defect & $5(13.5 \%)$ & $7(19.4 \%)$ & $0.5+$ \\
Complete IAS Defect & $1(2.7 \%)$ & $2(5.6 \%)$ & $0.5++$ \\
Halfmoon sign & $1(2.7 \%)$ & $1(2.8 \%)$ & $1++$ \\
Incomplete EAS Defect & $10(27 \%)$ & $7(19.4 \%)$ & $0.4+$ \\
Incomplete IAS Defect & 0 & $1(2.8 \%)$ & $0.3++$ \\
EAS thinning & $5(13.5 \%)$ & $3(8.3 \%)$ & $0.5++$ \\
IAS thinning & $5(13.5 \%)$ & $7(19.4 \%)$ & $0.4+$ \\
EAS thickening & 0 & 0 & \\
IAS thickening & $2(5.4 \%)$ & $3(8.3 \%)$ & $0.6++$ \\
\hline
\end{tabular}

Table 2 . Signs of EAS and IAS transperineal ultrasound evaluation. EAS; external anal sphincter, IAS; internal anal sphincter. 
Values are reported as number and percentage.

$+\mathrm{X}^{2}$ test

++ Fisher exact test.
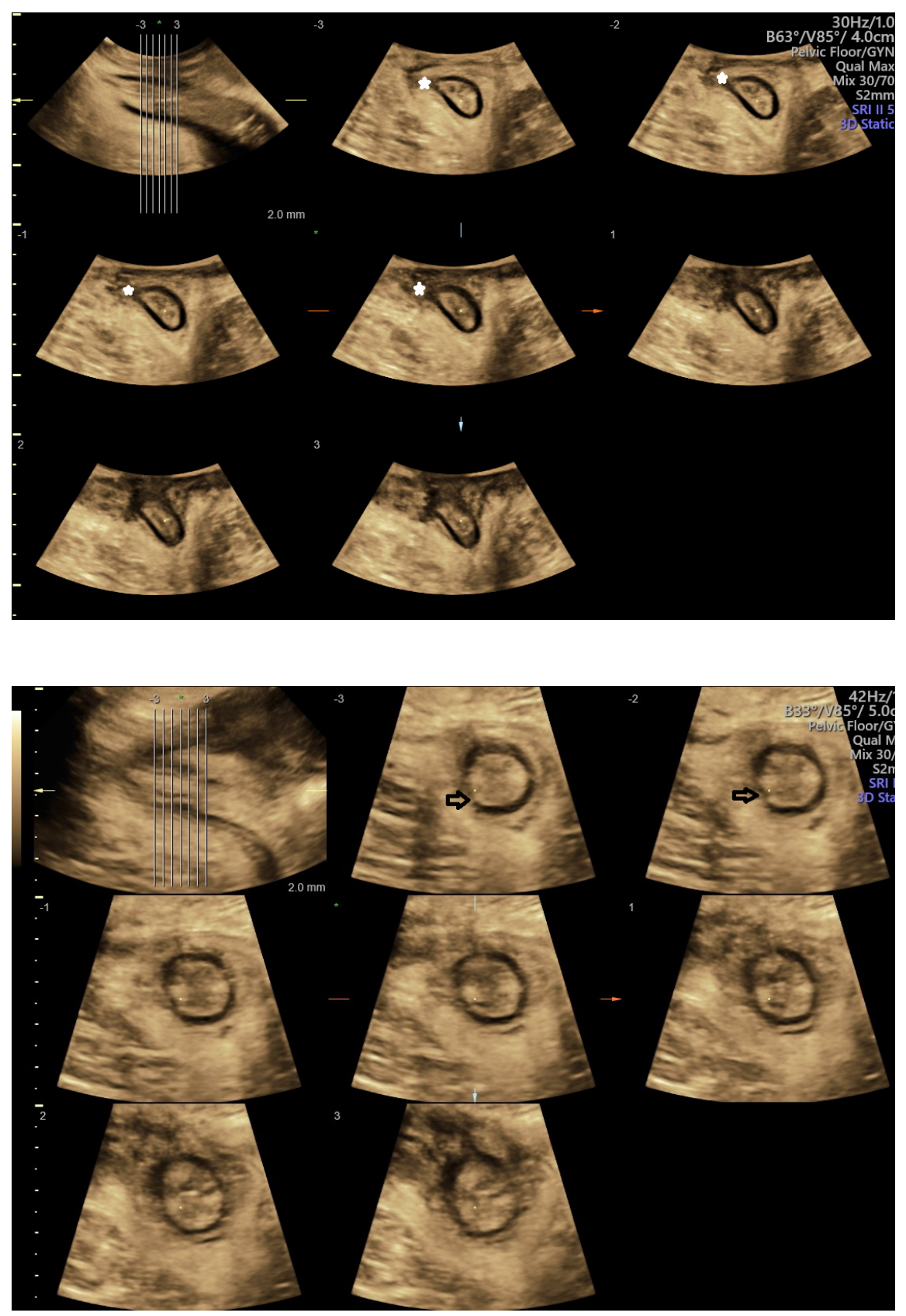


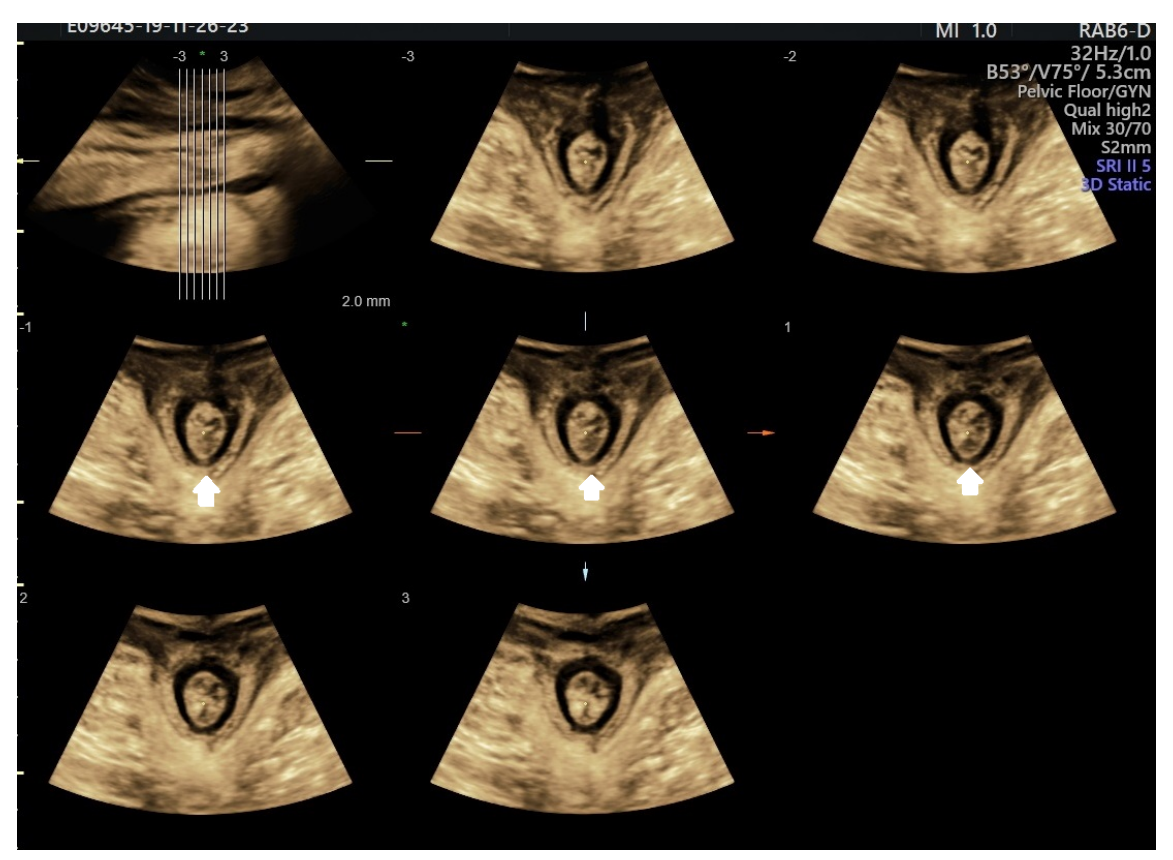

